\title{
ON THE DEFINING EQUATIONS OF HYPERSURFACE PURELY ELLIPTIC SINGULARITIES
}

\author{
NAOHIRO KANESAKA
}

(Received January 25, 2001, revised May 6, 2004)

\begin{abstract}
We investigate a class of isolated hypersurface singularities, the so-called purely elliptic singularities, of complex algebraic varieties of dimension greater than or equal to two. We show that, for hypersurface purely elliptic singularities defined by nondegenerate polynomials, Calabi-Yau varieties arising among the irreducible components of the essential divisors are concretely associated with the defining equations of these singularities, and that the birational class of the Calabi-Yau varieties does not depend on the irreducible components.
\end{abstract}

Introduction. A purely elliptic singularity is by definition a normal isolated singularity $(X, x)$ of an $r$-dimensional complex algebraic variety with $\delta_{m}(X, x)=1$ for any positive integer $m$, where $r \geq 2$ and $\delta_{m}(X, x)$ denotes the $m$-th $l^{2}$-pluri-genus of the normal isolated singularity (cf. [14]). Purely elliptic singularities can be thought of as higher dimensional analogues of simple elliptic singularities and cusps in dimension two. In general, for an isolated singularity $(X, x)$ of a complex algebraic variety, there exists a resolution of the singularity $\pi: \tilde{X} \rightarrow X$ such that the fibre $\pi^{-1}(x)_{\text {red }}$ is a divisor with simple normal crossings, which we call a good resolution of $(X, x)$. The exceptional set $E$ of a good resolution of a purely elliptic singularity has a characteristic complex analytic subset $E_{J}$ called the essential divisor [2], [3]; for example, in the case of a simple elliptic singularity, $E_{J}$ always consists of a nonsingular elliptic curve, while in the case of a cusp, $E_{J}$ consists of a cycle of nonsingular rational curves, which characterise the simple elliptic singularity and the cusp, respectively.

It was Ishii [2], [3] who first focused attention on the essential divisors of good resolutions of normal isolated $\boldsymbol{Q}$-Gorenstein singularities and unveiled their general algebrogeometric structures, by which we see that the essential divisors also have characteristic significance for purely elliptic singularities. In addition to this, Ishii-Watanabe [7] and Tomari [12] showed that Calabi-Yau varieties or Abelian varieties arise in general in the essential divisors of (partial) resolutions of purely elliptic singularities.

In contrast to these general results, our results in this paper is more explicit and summarised as follows. When purely elliptic singularities are hypersurface singularities defined by nondegenerate polynomials, the Calabi-Yau varieties, arising among the irreducible components of the essential divisors of good resolutions obtained by means of toric geometry, are concretely associated with the leading parts of the defining functions of the singularities, and the birational class of the Calabi-Yau varieties does not depend on the irreducible components. We note that a relationship between the leading parts of the defining functions of purely

2000 Mathematics Subject Classification. Primary 32S25; Secondary 14B05, 14M25. 
elliptic singularities and Calabi-Yau varieties is indicated in Yonemura [16] and Tomari [12], which motivated us to investigate this subject.

This paper is organised as follows. Section 1 is devoted to preliminaries and the statements of our results. We prove our main result in Section 2 with the help of a key lemma, which is proved in Section 3.

The author would like to express his gratitude to Professor Kimio Watanabe for his valuable comments and continuous stimulation during the preparation of this paper. He is also grateful to the referee for many thoughtful suggestions which improved the paper.

1. Preliminaries and results. 1.1. Preliminaries. First, we recall relevant definitions and known results about purely elliptic singularities. In what follows, a resolution $\pi: \tilde{X} \rightarrow X$ of a singularity $(X, x)$ is called a good resolution if the fibre $\pi^{-1}(x)_{\text {red }}$ is a divisor with simple normal crossings.

DEFINITION 1 (Watanabe [14]). A normal isolated singularity is called a purely elliptic singularity if $\delta_{m}(X, x)=1$ for any positive integer $m$. Here the $m$-th pluri-genus $\delta_{m}(X, x)$ is defined to be

$$
\begin{aligned}
\delta_{m}(X, x) & :=\operatorname{dim}_{C} \Gamma(X \backslash\{x\}, \mathcal{O}(m K)) / L^{2 / m}(X \backslash\{x\}) \\
& =\operatorname{dim}_{C} \Gamma(\tilde{X} \backslash E, \mathcal{O}(m K)) / \Gamma(\tilde{X}, \mathcal{O}(m K+(m-1) E)),
\end{aligned}
$$

where $\pi: \tilde{X} \rightarrow X$ is a good resolution of the singularity $(X, x)$ and $E$ is the reduced exceptional divisor. (We note that $\delta_{m}(X, x)$ is independent of the choice of a good resolution $\pi$ for every $m$.)

Definition 2 (Ishii [2], [3]). For a good resolution $\pi: \tilde{X} \rightarrow X$ of a normal isolated $Q$-Gorenstein singularity $(X, x)$, we define $K_{\tilde{X}}=\pi^{*} K_{X}+\sum_{i \in I} m_{i} E_{i}-\sum_{j \in J} m_{j} E_{j}$, where $m_{i}(i \in I)$ and $m_{j}(j \in J)$ are rational numbers, $m_{i} \geq 0$ for $i \in I, m_{j}>0$ for $j \in J$, and $E_{i}(i \in I)$ and $E_{j}(j \in J)$ are the irreducible components of $E=\pi^{-1}(x)_{\text {red. }}$. Denote by $\left[m_{j}\right]$ the integer such that $m_{j}-1<\left[m_{j}\right] \leq m_{j}$. The divisor $E_{J}:=\sum_{j \in J}\left[m_{j}\right] E_{j}$ is called the essential divisor.

The essential divisors characterise purely elliptic singularities in the following manner.

Proposition 1 (Ishii [2]). Suppose that $(X, x)$ is a normal isolated Gorenstein singularity. Then $(X, x)$ is a purely elliptic singularity if and only if the essential divisor of a good resolution is a reduced divisor.

In order to describe the configuration of the irreducible components of the essential divisor $E_{J}$, it is convenient to use the dual complex $\Gamma_{E_{J}}$ of $E_{J}$, which is a simplicial complex defined as follows:

(0) We associate a vertex with each irreducible component $E_{\alpha}$;

(1) If a pair of irreducible components $E_{\alpha}, E_{\beta}$ intersect, then we associate a line segment between the vertices corresponding to $E_{\alpha}, E_{\beta}$; 
(2) If three irreducible components $E_{\alpha}, E_{\beta}, E_{\gamma}$ intersect, we associate a triangle (twodimensional simplex) with the vertices corresponding to $E_{\alpha}, E_{\beta}, E_{\gamma}$; and

$(\mathrm{k}-1) \quad$ If $k$ irreducible components intersect, then we associate a $(k-1)$-dimensional simplex with the vertices corresponding to $E_{v_{1}}, E_{v_{2}}, \ldots, E_{v_{k}}$.

In [3], Ishii investigated more general periodically elliptic singularities. In that article, she introduced the notion of the "type" of a periodically elliptic singularity and classified $r$-dimensional periodically elliptic singularities into $r$ classes as follows: The type of a 1Gorenstein periodically elliptic singularity, namely a 1-Gorenstein purely elliptic singularity, is defined by the "Hodge type" of the cohomology group $H^{r-1}\left(E_{J}, \boldsymbol{C}\right)$ (see [3] for the precise definition). The type of a general periodically elliptic singularity is defined by the type of the purely elliptic singularity on its canonical cover. In addition, she gave a description of $\Gamma_{E_{J}}$ in connection with the types of periodically elliptic singularities, especially when the dimensions of the singularities are three. The following is only a part of her whole results on periodically elliptic singularities.

Proposition 2 (Ishii [5], [2]). Let $f: \tilde{X} \rightarrow X$ be a good resolution of an $r$ dimensional 1-Gorenstein purely elliptic singularity $(X, x)$. If $(X, x)$ is of type $(0, i)$, then $\operatorname{dim} \Gamma_{E_{J}} \geq r-1-i$. In particular, the equality holds in case $i=0$ or $r-1$.

Proposition 3 (Ishii [3]). Let $(X, x)$ be a three-dimensional Gorenstein purely elliptic singularity with the essential divisor $E_{J}$ of a good resolution $f: \tilde{X} \rightarrow X$. Then the essential divisor $E_{J}$ is as follows.

(1) If $(X, x)$ is of type $(0,2), E_{J}$ is an irreducible surface birationally equivalent to a K3-surface.

(2) If $(X, x)$ is of type $(0,1), E_{J}$ is a chain of surfaces $E_{1}, E_{2}, \ldots, E_{S}(s \geq 2)$ with rational surfaces $E_{1}, E_{S}$ and elliptic ruled surfaces $E_{2}, \ldots, E_{s-1}$, where $E_{i}$ and $E_{i+1}$ intersect along an elliptic curve for $i=1, \ldots, s-1$.

(3) If $(X, x)$ is of type $(0,0), E_{J}$ consists of rational surfaces with rational intersection curves and $\Gamma_{E_{J}}$ is a triangulation of the real two-dimensional sphere.

On the other hand, Tomari [12] investigated the canonical modifications of purely elliptic singularities by means of filtered blow-ups. In that work, he described a property of the exceptional set of the canonical modification of a purely elliptic singularity of type $(0, r-1)$ under the assumption of the existence of the canonical modification. Note that, in such a case, the exceptional set of the canonical modification is birationally equivalent to the essential divisor of any good resolution of the singularity.

PROPOSITION 4 (Tomari [12]). If an $r$-dimensional Gorenstein purely elliptic singularity of type $(0, r-1)$ has the canonical model, the exceptional set consists of an $(r-1)$ dimensional Calabi-Yau variety.

Here, we use the term Calabi-Yau variety in the following sense. 
DEFINITION 3. A complex normal irreducible $n$-dimensional projective algebraic variety $V$ with only Gorenstein canonical singularities is called a Calabi-Yau variety if $V$ has trivial canonical bundle and $H^{i}\left(V, \mathcal{O}_{V}\right)=0$ for $0<i<n$.

1.2. Problem and results. Among the results in the preceding subsection, what we would like to emphasise is that Calabi-Yau varieties arise in general in the essential divisors of (partial) resolutions of Gorenstein purely elliptic singularities if they are not of type $(0,0)$. Then our problem is to describe explicitly how it appears in special cases. We now consider the case where purely elliptic singularities are hypersurface singularities.

Let $(X, x)=(\{f=0\}, O)$ be an $r$-dimensional isolated hypersurface singularity defined by a polynomial

$$
f=\sum_{m_{0}, m_{1}, \ldots, m_{r} \geq 0} a_{m_{0}, m_{1}, \ldots, m_{r}} z_{0}^{m_{0}} z_{1}^{m_{1}} \cdots z_{r}^{m_{r}} \in \boldsymbol{C}\left[z_{0}, z_{1}, \ldots, z_{r}\right],
$$

where $O$ is the origin of $\boldsymbol{C}^{r+1}$ and $\boldsymbol{C}\left[z_{0}, z_{1}, \ldots, z_{r}\right]$ is a polynomial ring over the complex number field $\boldsymbol{C}$. Throughout this paper, we always assume that $r \geq 2$. We regard an ordered collection of indices $\mathbf{m}=\left(m_{0}, m_{1}, \ldots, m_{r}\right)$ of each monomial $z_{0}^{m_{0}} z_{1}^{m_{1}} \cdots z_{r}^{m_{r}}$ as an element of $M:=Z^{r+1}$, and write $\mathbf{z}^{\mathbf{m}}$ (resp. $a_{\mathbf{m}}$ ) for $z_{0}^{m_{0}} z_{1}^{m_{1}} \cdots z_{r}^{m_{r}}$ (resp. $a_{m_{0}, m_{1}, \ldots, m_{r}}$ ). We define $\Gamma_{+}(f)$ to be the convex hull of the union of the subsets $\mathbf{m}+\left(\boldsymbol{R}_{\geq 0}\right)^{r+1}$ of $M_{\boldsymbol{R}}:=M \otimes_{\mathrm{Z}} \boldsymbol{R} \cong$ $\boldsymbol{R}^{r+1}$ for all $\mathbf{m}$ such that $a_{\mathbf{m}} \neq 0$, and denote by $\Gamma(f)$ the union of the compact faces of $\Gamma_{+}(f)$. With each face $\gamma$ of $\Gamma(f)$, we associate a polynomial $f_{\gamma}(\mathbf{z})=\sum_{\mathbf{m} \in \gamma \cap M} a_{\mathbf{m}} \mathbf{z}^{\mathbf{m}}$. We say that $f$ is nondegenerate on $\gamma$ if $\partial f_{\gamma} / \partial z_{0}=\cdots=\partial f_{\gamma} / \partial z_{r}=0$ has no solution in $\left(C^{*}\right)^{r+1}$, and that $f$ is nondegenerate if $f_{\gamma}$ is nondegenerate on any face $\gamma$ of $\Gamma(f)$.

Here is a criterion for $(X, x)$ to be a purely elliptic singularity.

Proposition 5 (Watanabe [15]). Assume that $f$ is nondegenerate. Then $(X, x)=$ $(\{f=0\}, O)$ is a purely elliptic singularity if and only if $\mathbf{1}:=(1,1, \ldots, 1) \in \Gamma(f)$.

By Proposition 5, for a purely elliptic singularity defined by a nondegenerate polynomial $f$, there exists a unique face $\gamma_{\mathbf{1}}$ of $\Gamma(f)$ containing 1 in its relative interior. This face $\gamma_{\mathbf{1}}$ has a direct connection with the nature of the hypersurface purely elliptic singularity in many ways, which we will see in the following.

Tomari [12] showed that the weighted blow-up with respect to the unique weight associated with the face $\gamma_{\mathbf{1}}$ gives the canonical model of an $r$-dimensional hypersurface purely elliptic singularity of type $(0, r-1)$ if the defining function is nondegenerate. Therefore, as a corollary to Proposition 4, we have the following:

COROLlaRY 6 (Tomari [12]). The essential divisor $E_{J}$ of a good resolution of a hypersurface purely elliptic singularity of type $(0, r-1)$ is birational to an $(r-1)$-dimensional Calabi-Yau variety if the defining function is nondegenerate.

Now, we investigate in detail the essential divisor of a hypersurface purely elliptic singularity by means of toric geometry. We have a standard method using toric geometry to resolve a hypersurface isolated singularity defined by a nondegenerate polynomial as follows (see [13] 
for details): Let $N:=\operatorname{Hom}_{\boldsymbol{Z}}(M, \boldsymbol{Z})$ be the free $\boldsymbol{Z}$-module dual to $M$ and $N_{\boldsymbol{R}}:=N \otimes_{\boldsymbol{Z}} \boldsymbol{R}$ the scalar extension by $\boldsymbol{R}$. Since $M_{\boldsymbol{R}}$ and $N_{\boldsymbol{R}}$ are dual to each other as vector spaces over $\boldsymbol{R}$, an element $\mathbf{n}=\left(n_{0}, n_{1}, \ldots, n_{r}\right) \in N_{\boldsymbol{R}}$ gives rise to a family of parallel hyperplanes in $M_{\boldsymbol{R}}$. Denote by $\langle *, *\rangle$ the standard bilinear form on $M_{\boldsymbol{R}} \times N_{\boldsymbol{R}}$ and define $l(\mathbf{n}):=\min \left\{\langle\mathbf{m}, \mathbf{n}\rangle \mid \mathbf{m} \in \Gamma_{+}(f)\right\}$ for $\mathbf{n} \in N \cap\left(\boldsymbol{R}_{\geq 0}\right)^{r+1}$. For a face $\gamma$ of $\Gamma_{+}(f)$, define $\gamma^{*}:=\left\{\mathbf{n} \in\left(\boldsymbol{R}_{\geq 0}\right)^{r+1} \mid\langle\mathbf{m}, \mathbf{n}\rangle=\right.$ $l(\mathbf{n})$ for any $\mathbf{m} \in \gamma\}$. Then the set $\Sigma(f):=\left\{\gamma^{*} \mid \gamma \prec \Gamma_{+}(f)\right\}$ forms a finite subdivision of the positive orthant $\Sigma:=\left(\boldsymbol{R}_{\geq 0}\right)^{r+1}$, where $\gamma \prec \Gamma_{+}(f)$ means that $\gamma$ is a face of $\Gamma_{+}(f)$. Take a finite subdivision $\hat{\Sigma}(f)$ of $\Sigma(f)$ consisting of cones each of which is generated by a part of a basis of $N$. Then $\hat{\Sigma}(f)$ is also a subdivision of $\Sigma$. Dually, we obtain a proper, birational, $T_{N}$-equivariant morphism $\Pi: V_{\hat{\Sigma}(f)} \rightarrow V_{\Sigma} \cong C^{r+1}$ with $V_{\hat{\Sigma}(f)}$ a nonsingular variety. Denote by $\tilde{X}$ the proper transform of $X$ by $\Pi$. It is well-known that if the defining polynomial of $(X, x)$ is nondegenerate, then the restriction $\pi: \tilde{X} \rightarrow X$ of $\Pi$ to $\tilde{X}$ is a good resolution of the singularity $(X, x)$. In this paper, we call a good resolution of the singularity obtained in this way a toric good resolution for convenience.

The following proposition shows that the face $\gamma_{1}$ of $\Gamma(f)$ has topological information of the dual complex $\Gamma_{E_{J}}$.

Proposition $7([8])$. Let $(X, x)=(\{f=0\}, O)$ be an $r$-dimensional hypersurface purely elliptic singularity defined by a nondegenerate polynomial $f$. Then the dual complex $\Gamma_{E_{J}}$ of the essential divisor of a toric good resolution is isomorphic to a triangulation of the $\left(r-\operatorname{dim} \gamma_{\mathbf{1}}\right)$-dimensional sphere if $\operatorname{dim} \gamma_{\mathbf{1}} \geq 2$.

Furthermore, the leading polynomial $f_{\gamma_{\mathbf{1}}}=\sum_{\mathbf{m} \in \gamma_{\mathbf{1}} \cap M} a_{\mathbf{m}} \mathbf{z}^{\mathbf{m}}$ have algebro-geometric information about the intersections of the irreducible components corresponding to the definite parts of $\Gamma_{E_{J}}$. Indeed, from the polynomial $f_{\gamma_{1}}$ and the lattice $M_{\mathbf{1}}:=\boldsymbol{R}\left(\gamma_{\mathbf{1}}-\mathbf{1}\right) \cap M$, we can construct a $\left(\operatorname{dim} \gamma_{\mathbf{1}}-1\right)$-dimensional projective toric hypersurface, say $Y$, as we will see in Section 2.1, and we have the following:

PROPOSITION 8 ([8]). Under the assumption of Proposition 7, the intersection of the irreducible components of $E_{J}$ corresponding to a simplex of $\Gamma_{E_{J}}$ of maximal dimension is birational to $Y$, and every irreducible component of the essential divisor $E_{J}$ corresponding to a vertex contained in the interior of $\Gamma_{E_{J}}$ is birational to $Y{ }_{C_{C}} \boldsymbol{P}_{\boldsymbol{C}}^{r-\operatorname{dim} \gamma_{\mathbf{1}}}$.

Now, we state our main result, which shows that the polynomial $f_{\gamma_{1}}$ is concretely associated with Calabi-Yau varieties arising in $E_{J}$.

PROPOSITION 9. The variety $Y$ is birationally equivalent to a $\left(\operatorname{dim} \gamma_{1}-1\right)$-dimensional Calabi-Yau variety.

Combining Propositions 8 and 9, we obtain the following (cf. Propositions 3 and 4 and Corollary 6), which is our answer to the problem mentioned at the beginning of this subsection when a purely elliptic singularity is a hypersurface singularity defined by a nondegenetare polynomial. 
THEOREM 1. Assume that $E_{J}$ is the essential divisor of a toric good resolution of a hypersurface purely elliptic singularity defined by a nondegenerate polynomial $f$. Then every irreducible component of $E_{J}$ corresponding to a vertex contained in the interior of $\Gamma_{E_{J}}$ is birational to the product of a $\left(\operatorname{dim} \gamma_{\mathbf{1}}-1\right)$-dimensional Calabi-Yau variety and the $\left(r-\operatorname{dim} \gamma_{1}\right)$-dimensional projective space. Furthermore, the birational class of the CalabiYau variety is determined uniquely by the leading part $f_{\gamma_{1}}$ of $f$ and does not depend on the choice of an irreducible component.

2. Proof of the results. In this section, we prove the main result stated in the previous section. We state a key lemma i.e., Lemma 1 in Section 2.1 and prove Proposition 9 in Section 2.2.

2.1. Preparations for the proof of Proposition 9. Here, we recall some techniques from toric geometry and introduce a key lemma for the proof of Proposition 9. We refer the reader to [10] for general background on toric geometry and [1] for toric hypersurfaces.

First of all, we fix a compactification of hypersurfaces of algebraic tori defined by Laurent polynomials: Let $M$ be a free $Z$-module of $\operatorname{rank} n(\geq 2)$ and $N=\operatorname{Hom}_{Z}(M, Z)$ the $\boldsymbol{Z}$-module dual to $M$ as before. Denote by $\boldsymbol{C}[M]=\bigoplus_{\mathbf{m} \in M} \boldsymbol{C} \cdot \chi^{\mathbf{m}}$ the group algebra of $M$, namely, the Laurent polynomial ring. If an $n$-dimensional convex polyhedron $\Delta$ in $M_{\boldsymbol{R}}=M \otimes_{\mathrm{Z}} \boldsymbol{R}$ is given, we can compactify the algebraic torus $T_{N}=\operatorname{Spec} \boldsymbol{C}[M]$ to a projective toric variety as follows. For every $l$-dimensional face $\Theta$ of $\Delta$, denote by $\check{\sigma}(\Theta) \subset M_{\boldsymbol{R}}$ the convex $n$-dimensional cone consisting of all vectors $\lambda\left(\mathbf{p}-\mathbf{p}^{\prime}\right)$, where $\lambda \in \boldsymbol{R}_{\geq 0}, \mathbf{p} \in \Delta$ and $\mathbf{p}^{\prime} \in \Theta$. Let $\sigma(\Theta) \subset N_{\boldsymbol{R}}$ be the $(n-l)$-dimensional dual cone relative to $\check{\sigma}(\Theta)$. Then the set $\Sigma(\Delta)$ of all cones $\sigma(\Theta)$, where $\Theta$ runs over all faces of $\Delta$, determines a complete fan. As a result, we obtain a projective toric variety $\boldsymbol{P}_{\Delta}$ associated with $\Sigma(\Delta)$ containing $T_{N}$ as an open dense subset. A Laurent polynomial $f^{L}=\sum_{\mathbf{m} \in M} c_{\mathbf{m}} \chi^{\mathbf{m}} \in \boldsymbol{C}[M]$ defines a hypersurface $Z_{f^{L}, M}:=\left\{f^{L}=0\right\}$ in $T_{N}$. By taking its closure in $\boldsymbol{P}_{\Delta}$, a compactification $\overline{Z_{f^{L}, \Delta}}$ of $Z_{f^{L}, M}$ is obtained.

Next, let us recall the notion of $\Delta$-regularity for Laurent polynomials. Recall that there is a one-to-one correspondence between the orbits of the action of $T_{N}$ on $\boldsymbol{P}_{\Delta}$ and the faces of $\Delta$. By using this correspondence, $\boldsymbol{P}_{\Delta}$ can be written as a direct sum $\bigsqcup_{\Theta \prec \Delta} T_{\Theta}$, where $\Theta \prec \Delta$ means that $\Theta$ is a face of $\Delta$ and $T_{\Theta}$ denotes the orbit corresponding to a face $\Theta$ of $\Delta$. Then a Laurent polynomial $f^{L}$ is said to be $\Delta$-regular if for every face $\Theta \prec \Delta$, the variety $\overline{Z_{f}{ }^{L}, \Delta} \cap T_{\Theta}$ is an empty set or a smooth subvariety of codimension 1 in $T_{\Theta}$.

We denote by $\Delta\left(f^{L}\right)$ the Newton polyhedron of $f^{L}$, namely, the convex hull of the set $\left\{\mathbf{m} \in M_{\boldsymbol{R}} \mid c_{\mathbf{m}} \neq 0\right\}$ in $M_{\boldsymbol{R}}$.

Keeping these in mind, we now consider the projective variety $Y$ mentioned in Propositions 8 and 9. In fact, it is constructed as follows. Recall that we identify indices of monomials with elements of $M=Z^{r+1}$. Define $M_{1, \boldsymbol{R}}:=\boldsymbol{R}\left(\gamma_{\mathbf{1}}-\mathbf{1}\right)$, namely, the $\boldsymbol{R}$-vector subspace of $M_{R}$ generated by the subset $\gamma_{1}-1$, and set $M_{1}:=M_{1, R} \cap M$, which is a free $Z$-module of rank $i+1$. We define a Laurent polynomial $f_{\gamma_{1}}^{L}:=\sum_{\mathbf{m} \in \gamma_{1} \cap M} a_{\mathbf{m}} \chi^{\mathbf{m}-\mathbf{1}} \in \boldsymbol{C}\left[M_{\mathbf{1}}\right]$ for the defining polynomial $f=\sum_{\mathbf{m} \in M} a_{\mathbf{m}} \mathbf{z}^{\mathbf{m}} \in \boldsymbol{C}[\mathbf{z}]$ of the singularity. Then $Y$ is just the closure 
$\overline{Z_{f_{\gamma_{1}}^{L}, \Delta\left(f_{\gamma_{1}}^{L}\right)}}$ of the hypersurface $Z_{f_{\gamma_{1}}^{L}, M_{\mathbf{1}}} \subset \operatorname{Spec} \boldsymbol{C}\left[M_{\mathbf{1}}\right]$ in $\boldsymbol{P}_{\Delta\left(f_{\gamma_{1}}^{L}\right)}$. Proposition 9 claims that $Y$ is birational to a Calabi-Yau variety.

In order to state our key Lemma 1 below for proving Proposition 9, we give two definitions. Although these are weaker modifications of Batyrev's reflexive pairs for $n$-dimensional convex polyhedra in $M_{\boldsymbol{R}}$ and of $\Delta$-regularity for Laurent polynomials, they remain to be related to Calabi-Yau varieties as we will see in the lemma.

Definition 4 (cf. Batyrev [1, Definition 4.1.5]). Let $\Delta$ be an $n$-dimensional convex polyhedron in $M_{Q}$ containing the zero $\mathbf{0} \in M$ in its interior. Then the pair $(\Delta, M)$ is said to be $Q$-reflexive if the affine hyperplane generated by any $(n-1)$-dimensional face of $\Delta$ is of the form $\left\{\mathbf{m} \in M_{\boldsymbol{R}} \mid\langle\mathbf{m}, \mathbf{n}\rangle=-1\right\}$ for a primitive integral vector $\mathbf{n} \in N$.

Definition 5 (cf. Batyrev [Definition 3.1.1]). Assume that $(\Delta, M)$ is a $\boldsymbol{Q}$-reflexive pair and that $f^{L} \in \boldsymbol{C}[M]$ is a Laurent polynomial with $\Delta\left(f^{L}\right) \subset \Delta$. We say that $f^{L}$ is quasi-regular with respect to $\Delta$ if $\Delta\left(f^{L}\right)$ is $n$-dimensional and contains the zero $\mathbf{0} \in M$ in its interior and $f^{L}$ is $\Delta\left(f^{L}\right)$-regular.

LEMMA 1. Let $(\Delta, M)$ be a $Q$-reflexive pair. If $f^{L}$ is quasi-regular with respect to $\Delta$, then $\overline{Z_{f L}, \Delta}$ is birational to a $(\operatorname{dim} \Delta-1)$-dimensional Calabi-Yau variety in the sense of Definition 3.

We prove this lemma in Section 3 and we prove Proposition 9 with the help of this lemma.

2.2. Proof of Proposition 9. We show that there exists a compact convex polyhedron $\Delta_{1}^{\prime}$ such that $\left(\Delta_{1}^{\prime}, M_{1}\right)$ is a $\boldsymbol{Q}$-reflexive pair and that $f_{\gamma_{1}}^{L}$ is quasi-regular with respect to $\Delta_{\mathbf{1}}^{\prime}$. Then the proposition is proved by Lemma 1, since the closures of $Z_{f_{\gamma_{1}}^{L}, M_{\mathbf{1}}}$ in $\boldsymbol{P}_{\Delta_{\mathbf{1}}}$ and in $\boldsymbol{P}_{\Delta_{\mathbf{1}}^{\prime}}$ are birational to each other.

Now, assume that $\operatorname{dim}_{R} \gamma_{\mathbf{1}}=r-k$. Then there exist exactly $k+1$ compact faces $\delta^{(0)}, \delta^{(1)}, \ldots, \delta^{(k)}$ of $\Gamma(f)$ such that $\operatorname{dim}_{\boldsymbol{R}} \delta^{(s)}=r$ for any $s(0 \leq s \leq k)$ and $\gamma_{\mathbf{1}}=$ $\delta^{(0)} \cap \delta^{(1)} \cap \ldots \cap \delta^{(k)}$. Let $H^{(0)}, H^{(1)}, \ldots, H^{(k)}$ be the hyperplanes of $M_{\boldsymbol{R}}$ spanned by $\delta^{(0)}, \delta^{(1)}, \ldots, \delta^{(k)}$, respectively. Then we have $\gamma_{\mathbf{1}} \subset\left(\boldsymbol{R}_{\geq 0}\right)^{r+1} \cap\left(\bigcap_{s=0}^{k} H^{(s)}\right)$. We now define $\Delta_{\mathbf{1}}^{\prime}:=\left(\boldsymbol{R}_{\geq 0}\right)^{r+1} \cap\left(\cap_{s=0}^{k} H^{(s)}\right)-\mathbf{1}$.

Claim. The pair $\left(\Delta_{\mathbf{1}}^{\prime}, M_{\mathbf{1}}\right)$ is $\boldsymbol{Q}$-reflexive and $f_{\gamma_{1}}^{L}$ is quasi-regular with respect to $\Delta_{\mathbf{1}}^{\prime}$.

PROOF. The first part of the claim is proved in [8]. Thus we prove the second part. As we saw before, $\gamma_{1}-\mathbf{1} \subset \Delta_{1}^{\prime}$ and $\operatorname{dim} \gamma_{1}-\mathbf{1}=\operatorname{dim} \Delta_{1}^{\prime}=\operatorname{dim} M_{1, R}$ hold. Note that $\gamma_{1}-\mathbf{1}$ is the Newton polyhedron of $f_{\gamma_{1}}^{L}$ and that $\gamma_{1}-\mathbf{1}$ contains the zero $\mathbf{0}$ in its interior by the definition of $\gamma_{\mathbf{1}}$. The nondegeneracy of the defining polynomial $f$ guarantees the $\Delta\left(f_{\gamma_{1}}^{L}\right)$-regularity of $f_{\gamma_{1}}^{L}$.

\section{Proof of Lemma 1.}

3.1. Proof of Lemma 1. Let us prove Lemma 1. By Batyrev [1, Proposition 2.2.19], there exists a complete fan $\Sigma_{0}$ with the following properties: (i) $\Sigma_{0}$ is simplicial, (ii) $\Sigma_{0}(1)=$ 
$\Sigma(\Delta)(1)$, where $\Sigma_{0}(1)$ (resp. $\left.\Sigma(\Delta)(1)\right)$ denotes the set of one-dimensional cones in $\Sigma_{0}$ (resp. $\Sigma(\Delta)$ ), and (iii) the toric variety $V_{\Sigma_{0}}$ associated with $\Sigma_{0}$ is projective. Note that the condition (i) implies that $V_{\Sigma_{0}}$ has at worst $\boldsymbol{Q}$-factorial log-terminal singularities. Let $Y_{0}$ be the closure of $Z_{f^{L}, M}$ in $V_{\Sigma_{0}}$. Then it is birational to $\overline{Z_{f^{L}, \Delta}}$. We show that $Y_{0}$ is a Calabi-Yau variety by proving the following Lemmas 2, 3 and 4.

LEMMA 2. $Y_{0}$ is an anticanonical divisor of $V_{\Sigma_{0}}$, that is, $Y_{0}+K_{V_{\Sigma_{0}}} \sim 0$.

LEMMA 3. $Y_{0}$ is a normal projective variety with at worst canonical singularities.

LEMMA 4. $H^{k}\left(Y_{0}, \mathcal{O}_{Y_{0}}\right)=0$ for $0<k<n-1$.

Indeed, it follows that $Y_{0}$ is a Calabi-Yau variety from these lemmas. By Lemma 3, $Y_{0}$ is a normal irreducible projective variety with at worst canonical singularities. Then $K_{Y_{0}}$ is a Cartier divisor in codimension two, and hence the adjunction formula $K_{Y_{0}}=\left.\left(K_{V_{\Sigma_{0}}}+Y_{0}\right)\right|_{Y_{0}}$ holds (see Kollár-Mori [9, Corollary 5.18 and Remark 5.47]). Hence, by Lemma 2, $K_{Y_{0}}$ is linearly equivalent to zero, that is, the canonical bundle of $Y_{0}$ is trivial. Therefore, all the singularities on $Y_{0}$ are rational and Gorenstein. In addition, by Lemma $4, H^{k}\left(Y_{0}, \mathcal{O}_{Y_{0}}\right)=0$ for $0<k<n-1$. Hence $Y_{0}$ is a Calabi-Yau variety.

Proof of Lemma 2. For a Laurent polynomial $f^{L}=\sum_{\mathbf{m} \in M} c_{\mathbf{m}} \chi^{\mathbf{m}} \in \boldsymbol{C}[M]$ and an integral vector $\mathbf{p} \in N$, define $\mathbf{p}\left(f^{L}\right):=\min \left\{\langle\mathbf{m}, \mathbf{p}\rangle \mid c_{\mathbf{m}} \neq 0\right\}$. Then we have $\operatorname{div}_{V_{\Sigma_{0}}}\left(f^{L}\right)=$ $Y_{0}+\sum_{\rho \in \Sigma_{0}(1)} \mathbf{n}_{\rho}\left(f^{L}\right) \cdot D_{\rho}$, where $\operatorname{div}_{V_{\Sigma_{0}}}\left(f^{L}\right)$ denotes the principal divisor of $f^{L}$ on $V_{\Sigma_{0}}$ and $\mathbf{n}_{\rho}$ is the primitive integral generator of $\rho$.

Take a one-dimensional cone $\rho$ in $\Sigma_{0}$. Then, since $\Sigma_{0}(1)=\Sigma(\Delta)(1)$, the half-space $\left\{\mathbf{m} \in M_{Q} \mid\left\langle\mathbf{m}, \mathbf{n}_{\rho}\right\rangle \geq-1\right\}$ contains $\Delta$, and the intersection of the boundary of the half-space and $\Delta$ is a codimension-one face of $\Delta$. Since $f^{L}$ is quasi-regular with respect to $\Delta$ and the Newton polyhedron $\Delta\left(f^{L}\right) \subset \Delta$ is an integral polyhedron containing the zero $\mathbf{0} \in M$ in its interior, there exists at least one integral point $\mathbf{m}$ with $c_{\mathbf{m}} \neq 0$ on each codimension-one face of $\Delta$. Therefore $\mathbf{n}_{\rho}\left(f^{L}\right)=-1$ holds.

Consequently, we have $\operatorname{div}_{V_{\Sigma_{0}}}\left(f^{L}\right)=Y_{0}+\left(-\sum_{\rho \in \Sigma_{0}(1)} D_{\rho}\right)=Y_{0}+K_{V_{\Sigma_{0}}}$.

In order to prove Lemmas 3 and 4, let us define another compactification $Y_{1}$ of $Z_{f^{L}, M}$ and birational morphisms $W \rightarrow Y_{0}$ and $W \rightarrow Y_{1}$ from a nonsingular variety $W$ as follows: Define $Y_{1}$ to be the closure of $Z_{f^{L}, M}$ in $\boldsymbol{P}_{\Delta\left(f^{L}\right)}$. We note that $Y_{1}$ is an ample divisor of $\boldsymbol{P}_{\Delta\left(f^{L}\right)}$. Put $\Sigma_{1}:=\Sigma\left(\Delta\left(f^{L}\right)\right)$ and take a finite common subdivision $\hat{\Sigma}$ of $\Sigma_{0}$ and $\Sigma_{1}$ consisting of cones each of which is generated by a part of a basis of $M$. Then we obtain a complete nonsingular variety $V_{\hat{\Sigma}}$, and proper birational morphisms $\Pi_{0}: V_{\hat{\Sigma}} \rightarrow V_{\Sigma_{0}}$ and $\Pi_{1}: V_{\hat{\Sigma}} \rightarrow V_{\Sigma_{1}}$. Let $W$ be the proper transform of $Y_{1}$ with respect to $\Pi_{1}$. Then $\pi_{1}:=\left.\Pi_{1}\right|_{W}: W \rightarrow Y_{1}$ is a resolution of singularities, since $f^{L}$ is $\Delta\left(f^{L}\right)$-regular. From this, it follows that $W$ is also the proper transform of $Y_{0}$ with respect to $\Pi_{0}$, and that $\pi_{0}:=\left.\Pi_{0}\right|_{W}: W \rightarrow Y_{0}$ is also a resolution of singularities.

Proof of Lemma 3. The following lemma due to Ishii is of essential use in our argument. 
Proposition 10 (Ishii [4, Lemma 2.7]). Let $Y \subset Z$ be an irreducible Weil divisor on a variety $Z$. Assume that $Z$ admits at worst $Q$-factorial log-terminal singularities. Let $\psi: \tilde{Y} \rightarrow Y$ be a resolution of singularities on $Y$. Assume that

$$
K_{\tilde{Y}}=\psi^{*}\left(\left.\left(K_{Z}+Y\right)\right|_{Y}\right)+\sum_{i} m_{i} E_{i}
$$

with $m_{i}>-1$ for all $i$, where $E_{i}$ 's are the irreducible components of the exceptional divisor of $\psi$. Then $Y$ is normal, and $Y$ has at worst log-terminal singularities. In particular, if $m_{i} \geq 0$ for all $i$, then $Y$ has at worst canonical singularities.

Recall that $V_{\Sigma_{0}}$ has at worst $\boldsymbol{Q}$-factorial log-terminal singularities. On the other hand, as was seen in the proof of Lemma 2, $Y_{0}+K_{V_{\Sigma_{0}}}=\operatorname{div}_{V_{\Sigma_{0}}}\left(f^{L}\right)$ holds. Because of this, we obtain

$$
\begin{aligned}
\Pi_{0}^{*}\left(Y_{0}+K_{V_{\Sigma_{0}}}\right) & =\Pi_{0}^{*} \operatorname{div}_{V_{\Sigma_{0}}}\left(f^{L}\right)=\operatorname{div}_{V_{\hat{\Sigma}}}\left(\Pi_{0}^{*} f^{L}\right) \\
& =W+\sum_{\hat{\rho} \in \hat{\Sigma}(1)} \mathbf{n}_{\hat{\rho}}\left(f^{L}\right) \cdot D_{\hat{\rho}} \\
& =\left(W+K_{V_{\hat{\Sigma}}}\right)+\sum_{\hat{\rho} \in \hat{\Sigma}(1) \backslash \Sigma_{0}(1)}\left(\mathbf{n}_{\hat{\rho}}\left(f^{L}\right)+1\right) \cdot D_{\hat{\rho}},
\end{aligned}
$$

and hence

$$
K_{V_{\hat{\Sigma}}}+W=\Pi_{0}^{*}\left(K_{V_{\Sigma_{0}}}+Y_{0}\right)+\sum_{\hat{\rho} \in \hat{\Sigma}(1) \backslash \Sigma_{0}(1)}\left(-\mathbf{n}_{\hat{\rho}}\left(f^{L}\right)-1\right) \cdot D_{\hat{\rho}} .
$$

Since the Newton polyhedron $\Delta\left(f^{L}\right)$ of $f^{L}$ contains the zero $\mathbf{0} \in M$ in its interior, we have

$$
\mathbf{n}_{\hat{\rho}}\left(f^{L}\right)=\min \left\{\left\langle\mathbf{m}, \mathbf{n}_{\hat{\rho}}\right\rangle \mid c_{\mathbf{m}} \neq 0\right\} \leq-1,
$$

and hence $-\mathbf{n}_{\hat{\rho}}\left(f^{L}\right)-1 \geq 0$ for any $\hat{\rho} \in \hat{\Sigma}(1) \backslash \Sigma_{0}(1)$. Therefore, by the adjunction formula, we obtain

$$
K_{W}=\left.\left(K_{V_{\hat{\Sigma}}}+W\right)\right|_{W}=\pi_{0}^{*}\left(\left.\left(K_{V_{\Sigma_{0}}}+Y_{0}\right)\right|_{Y_{0}}\right)+\sum_{i} \beta_{i} \cdot E_{i}
$$

with $\beta_{i} \geq 0$. Hence, by Proposition $10, Y_{0}$ is normal and has at worst canonical singularities. Since $V_{\Sigma_{0}}$ is projective, so is $Y_{0}$.

Proof of Lemma 4. Recall that the other compactification $Y_{1}$ of $Z_{f^{L}, M}$ in $\boldsymbol{P}_{\Delta\left(f^{L}\right)}$ is an ample divisor of $\boldsymbol{P}_{\Delta\left(f^{L}\right)}$. By the vanishing theorem for arbitrary ample divisors on toric varieties, we have $H^{k}\left(Y_{1}, \mathcal{O}_{Y_{1}}\right)=0$ for $0<k<n-1$. Moreover, by Batyrev [1, Corollary 3.1.6], every singularity on $Y_{1}$ is analytically equivalent to a toric singularity, so that it is a rational singularity.

Since $Y_{0}$ (resp. $Y_{1}$ ) has at worst rational singularities and $\pi_{0}: W \rightarrow Y_{0}$ (resp. $\pi_{1}: W \rightarrow$ $Y_{1}$ ) is a resolution of singularities, we have $R^{k} \pi_{0 *} \mathcal{O}_{W}=0$ (resp. $R^{k} \pi_{1 *} \mathcal{O}_{W}=0$ ) for $0<k$. 
Hence, for $0<k$,

$$
\begin{aligned}
& H^{k}\left(W, \mathcal{O}_{W}\right) \cong H^{k}\left(Y_{0}, \pi_{0 *} \mathcal{O}_{W}\right)=H^{k}\left(Y_{0}, \mathcal{O}_{Y_{0}}\right) \\
&\text { (resp. } \left.\quad H^{k}\left(W, \mathcal{O}_{W}\right) \cong H^{k}\left(Y_{1}, \pi_{1 *} \mathcal{O}_{W}\right)=H^{k}\left(Y_{1}, \mathcal{O}_{Y_{1}}\right)\right) .
\end{aligned}
$$

Therefore, we obtain $H^{k}\left(Y_{0}, \mathcal{O}_{Y_{0}}\right)=0$ for $0<k<n-1$.

\section{REFERENCES}

[1] V. V. BATYREv, Dual polyhedra and mirror symmetry for Calabi-Yau hypersurfaces in toric varieties, J. Algebraic Geom. 3 (1994), 493-535.

[ 2 ] S. ISHII, On isolated Gorenstein singularities, Math. Ann. 270 (1985), 541-554.

[ 3 ] S. IsHII, Isolated $\boldsymbol{Q}$-Gorenstein singularities of dimension three, Complex Analytic Singularities (T. Suwa and P. Wagreich editors), 165-198, Adv. Stud. in Pure Math., 8, Kinokuniya, Tokyo and North-Holland, Amsterdam, New York and Oxford, 1986.

[ 4 ] S. IsHII, The canonical modifications by weighted blowups, J. Algebraic Geom. 5 (1996), 783-799.

[ 5 ] S. IsHII, Introduction to singularities (in Japanese), Springer-Verlag Tokyo, Tokyo, 1997.

[6] S. IsHII, Minimal, canonical and log-canonical models of hypersurface singularities, Birational algebraic geometry (Baltimore, MD, 1996), 63-77, Contemp. Math., 207, Amer. Math. Soc., Providence, RI, 1997.

[ 7 ] S. Ishit And K. WATANABE, A geometric characterisation of a simple $K 3$ singularity, Tôhoku Math. J. 44 (1992), 19-24.

[ 8 ] N. Kanesaka, Explicit structures of three-dimensional hypersurface purely elliptic singularities of type $(0,1)$, Tsukuba J. Math. 25 (2001), 47-73.

[9] J. Kollár And S. MoRI, Birational Geometry of Algebraic Varieties, Cambridge Tracts in Math., 134, Cambridge University Press, Cambridge, 1998.

[10] T. OdA, Convex bodies and algebraic geometry, Ergeb. Math. Grenzgeb. (3), 15, Springer-Verlag, Berlin, Heidelberg, New York, London, Paris, Tokyo, 1988.

[11] M. TOMARI AND K.-I. WATANABE, Filtered rings, filtered blowing-ups and normal two-dimensional singularities with "star-shaped" resolution, Publ. Res. Inst. Math. Sci. 25 (1989), 681-740.

[12] M. TOMARI, The canonical filtration of higher dimensional purely elliptic singularity of a special type, Invent. Math. 104 (1991), 497-520.

[13] A. N. VARChENKo, Zeta-function of monodromy and Newton's diagram, Invent. Math. 37 (1976), 253-262.

[14] K. Watanabe, On plurigenera of normal isolated singularities I, Math. Ann. 250 (1980), 65-94.

[15] K. WatAnABE, On plurigenera of normal isolated singularities II, Complex Analytic Singularities (T. Suwa and P. Wagreich editors), 671-685, Adv. Stud. in Pure Math., 8, Kinokuniya, Tokyo and North-Holland, Amsterdam, New York and Oxford, 1986.

[16] T. YonemuRA, Hypersurface simple $K 3$ singularities, Tôhoku Math. J. 42 (1990), 351-380.

DEPARTMENT OF MATHEMATICS

TOYota NATional COLLEge OF TeChNOLOGY

2-1, EISEICHO, TOYOTA 471-8525

JAPAN

E-mail address: kanesaka@toyota-ct.ac.jp 